\title{
Time Domain Double-Loaded Electromagnetic Field Probe applied to Unmanned Air Vehicles
}

\author{
Marc Pous ${ }^{1}$, Sergio Fernández ${ }^{2}$, Manuel Añón ${ }^{2}$, Miguel R. Cabello ${ }^{3}$, Luis D. Angulo ${ }^{3}$, Ferran Silva ${ }^{1}$ \\ ${ }^{1}$ Grup de Compatibilitat Electromagnètica (GCEM), Departament d'Enginyeria Electrònica (DEE) \\ Universitat Politècnica de Catalunya (UPC) Barcelona, Spain \\ ${ }^{2}$ Aerospace Technique National Institute (INTA), EMC Area, Madrid, Spain \\ ${ }^{3}$ Dept. Electromagnetismo y F. M. Facultad de Ciencias Universidad de Granada, Granada, Spain \\ marc.pous@upc.edu
}

\begin{abstract}
In this study, measurements inside the fuselage of an unmanned air vehicle (UAV) are conducted using a novel double-loaded loop probe. The characterization and evaluation of electromagnetic interference (EMI) within UAVs is a main research topic and requires state-of-the-art measurement methodologies. The developed probe is capable of obtaining accurate measurements as commercial reference E-Field broadband probes, introducing new advantages based on the reduction of the probe's electronics and the availability of the full time-domain data. Allowing to compute simultaneously the electric and the magnetic field and to measure high-amplitude electromagnetic pulses. The laser-probe works together with multi-channel full-time domain system computing the electromagnetic fields using an oscilloscope. Regarding the double-loaded loop probe characterization and validation, it has been carried out by comparing the results with different validation methods, like Feature Selective Validation Method and the Integrated Error against Logarithmic Frequency.
\end{abstract}

Keywords-Time-domain measurements, Electromagnetic interferences, Feature Selective Validation, unmanned aircraft systems

\section{INTRODUCTION}

Electromagnetic Interference (EMI) is a major concern for the development of Unmanned Air Vehicles (UAVs). Particularly, since UAVs are constructed with composite materials, which are not as effective in shielding compared with previous metallic materials. Therefore, the Electromagnetic Fields (EMF) produced by different sources like lightning, High Electromagnetic Pulses (HEMP) or HighIntensity Radiated Field (HIRF) inside the UAV is a crucial matter in terms of Electromagnetic Compatibility (EMC) and it is studied in many research projects [1-2].

The proper characterization and measurement of EMF allow manufacturing UAVs capable of managing EMI according to the state-of-the-art [3]. Being capable of facing up Electromagnetic Interference (EMI) coupled within the composite structure of the UAV, where the EMF components are far away from the free-space approximation. Thus, it is needed to place EMF probes within UAVs but at the same time ensuring that the placement of the probe disturbs slightly the EM-fields. EM simulation is a common tool to overcome probe placement computing the EM field inside UAVs or other aeronautical applications. However, model with sufficient detail the complexity of materials and merging parts is a huge challenge. Therefore, this work is still going on within the framework of many international research projects [1-2].

Alternatively, the employment of small fiber optic (FO) powered probes is available at the market. These Electric Field (E-Field) or magnetic Field (H-Field) probes are largely used in EMC testing. However, these are broadband probes and they are measuring the contribution of the full spectrum at the same time. Making not feasible to distinguish frequency components as the user only gets a single value of the entire working frequency range of the probe. Other commercial probes options, like EM dot sensor which measures the time derivative of the electric displacement (D-dot), allow us to distinguish the information in the frequency domain. Nevertheless, these probes are suitable for far-field conditions and the metallic structure of the probe can produce distortion when it is used with the coaxial cables inside the small cavities of UAVs. Moreover, we only obtain the data from one of the components of the electric field, omitting the three-axes of the EMF.

Consequently, it is necessary to create a tailor-made probe considering the final application. A common situation required to characterize EMF inside UAVs, is to develop a probe capable of measuring the full spectrum with a single measurement. Measuring broadband disturbances like impulsive noise produced by transients, but at the same time to have the capability to quantify the EH-Fields at certain frequencies. For instance, to evaluate the full broadband contribution of HEMP or a particular frequency range where susceptible communication systems are allocated.

As aforementioned, it is also important to reduce the size and electronics of the EMF tailor-made probe in order to not influence the propagation of the fields within the cavity. The conception of the developed probe explained in section II.A is to convert the electrical signal coupled at the double-loaded loop probe to an optical one and after apply the post-processing needed. Hence, we will have available the time-domain data to perform Full Time Domain measurements [4-5]. In the next section, the EMF probe and the post-processing stage using a multi-channel Time-Domain system is explained. In section III, measurement results of a UAV conducted at INTA in Madrid 
are shown. Placing the tailor-made probe inside the composite small structure of the UAV, and comparing the results with other commercial probes used as reference.

\section{EMF DEVELOPED PROBE}

\section{A. EMF double-loaded loop Probe}

A double-loaded loop probe is constructed by a loop with two gaps at opposite sides that are both loaded with identical loads. The probe is based on the theory presented by King in 1969 [6], as well as on the later developments of Kanda and Wieckowski in 1980s [7], [8]. The presence of an electromagnetic field induces a current within the loop, which is the contribution of the electric field and the magnetic field. Therefore, it is equivalent to consider that the response of the double-loaded loop is the superposition of an electric dipole and a magnetic loop. When the loop is electrically small, the current at each load placed at opposite sides (that is, $\varphi_{1}$ and $\varphi_{2}$ $\left.=\varphi_{1}+\pi\right)$, can be expressed as $[7,8]$

$$
\begin{aligned}
& I\left(\varphi_{1}\right)=\frac{-j \omega \cdot \pi \cdot b^{2} \cdot F_{H}}{1+2 Y_{0} Z_{L}} \cdot H-\frac{2 \pi \cdot b \cdot F_{E}}{1+2 Y_{1} Z_{L}} \cdot E \\
& I\left(\varphi_{2}\right)=\frac{-j \omega \cdot \pi \cdot b^{2} \cdot F_{H}}{1+2 Y_{0} Z_{L}} \cdot H+\frac{2 \pi \cdot b \cdot F_{E}}{1+2 Y_{1} Z_{L}} \cdot E
\end{aligned}
$$

where: $b$ is the radius of the loop, $\varphi_{1}$ and $\varphi_{2}=\varphi_{1}+\pi$ define the angular position in the loop, $H$ is the amplitude of the magnetic field, $E$ is the amplitude of the electric field, $F_{E}$ and $F_{H}$ are functions depending on the direction of the fields, $Y_{0}$ and $Y_{1}$ are the first two terms of the admittance of the loop expressed as a series expansion, and $Z_{L}$ is the load at each gap.

The half addition and the half subtraction of those equations enable us to isolate the contribution of each field:

$$
\begin{aligned}
& I_{\Sigma}=\frac{-j \omega \cdot \pi \cdot b^{2} \cdot F_{H}}{1+2 Y_{0} Z_{L}} \cdot H \\
& I_{\Delta}=\frac{2 \pi \cdot b \cdot F_{E}}{1+2 Y_{1} Z_{L}} \cdot E
\end{aligned}
$$

The addition is directly related to the magnetic field and the subtraction is associated with the electric field. In previous developments [9-11], the addition and subtraction were carried out at the electronics attached to the Printed Circuit Board (PCB) probe. Currently, the probe is a $10 \mathrm{~cm}$ double-loaded loop implemented on a PCB and the loads are the intrinsic impedance of the transmitting lasers (Fig. 1). Otherwise, in this study, the operations are not performed at the probe's electronics and this is an important innovation. The current induced at each of the lasers is used as the information and transmitted to a detector circuitry directly connected to an oscilloscope. Using two channels of the oscilloscope to acquire the time-domain data and after compute the EMF using a Personal Computer (PC), as it is explained in section B. Consequently, all the mathematical processing is done with the PC and not at the probe's electronics as it was done before. Finally, regarding the double-loaded loop probe developed, the lasers have a maximum bandwidth of $2.5 \mathrm{GHz}$ and the probe is powered with a $9 \mathrm{~V}$ battery through a voltage regulator.

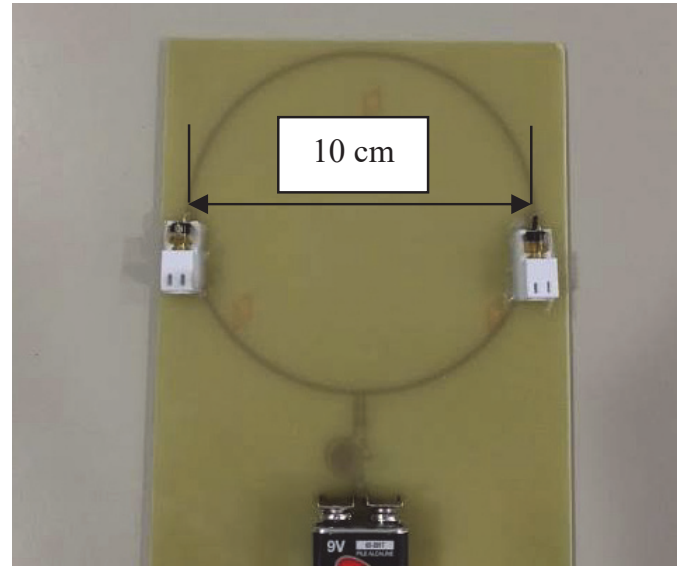

Fig. 1. Picture of the developed double-loaded loop probe.

\section{B. Post-processing (TD data)}

As it is described in the previous section, the lasers of the EMF probe are transmitting the loop's current through the FO link at the laser-loads connected directly to the oscilloscope. Receiving the signal synchronously at both channels of the Tektronix DPO5104B oscilloscope.

The measurement system employed is based on the Full Time Domain EMI measurement system developed by GCEMUPC [4-5], [12-14]. Using time-domain data acquired by an oscilloscope to compute EMI measurements; the basic block diagram of the measurement system is shown in Fig. 2.

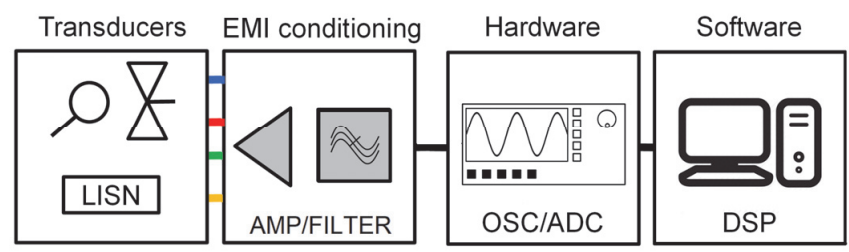

Fig. 2. Block diagram of the Full TDEMI measurement system.

In our application, in order to improve the accuracy of the results, we apply oversampling and signal averaging, which is possible as we want to measure sinewave. Once we have available the time-domain signal, we are capable of postprocessing the information using mathematical software like MATLAB. Evidently, with the capacity to add or subtract the in-phase signals of both lasers, which is the key-point to find out the EMF through a final Fast Fourier Transform (FFT). Furthermore, the possibilities are open to obtain broadband data or results for a single frequency as we have all the timedomain data just limited by the bandwidth of the oscilloscope. 
Finally, it is necessary to apply a conversion factor for the double-loop probe in order to relate the measured voltage with the EH-Fields. This step is done with a characterization procedure employing a reference commercial probe.

\section{Characterization of the double-load loop probe}

The study is focused on a final application which intends to measure only the E-Field, thus, the characterization is done through a reference E-Field probe. The probe selected is an Amplifier Research (AR) probe model FL7006 capable of measuring the three-axis E-Field with an accuracy of $0.8 \mathrm{~dB}$ between $100 \mathrm{kHz}$ and $1 \mathrm{GHz}$. Otherwise, an extensive work on calibrating the double-load loop probe will be required in the future, if validation results are confident.

The characterization is done generating an E-Field inside a Full Anechoic Chamber (FAC). The electric field is produced by a Bilog Antenna model CBL6143, connected to the output of an AR amplifier model AR150W1000 and a Rohde\&Schwarz Radio Frequency (RF) generator model SML03. The antenna is polarized vertically and the distance between the antenna and the reference probe is $2 \mathrm{~m}$. In these conditions, a target field of $5 \mathrm{~V} / \mathrm{m}$ is calibrated, afterward, the reference probe is substituted by the developed probe. The position of the probe is set according to receive the maximum electric field and the minimum magnetic field. Therefore, the double-load loop is placed with the lasers at the same height and the loop faced to the antenna, perpendicular to the propagation axis of the EM wave. The EH-Field probe is characterized by measuring simultaneously the voltage at both channels of the oscilloscope, between $100 \mathrm{MHz}$ and $1 \mathrm{GHz}$. Fig. 3 shows the TD data acquired when a field of $5 \mathrm{~V} / \mathrm{m}$ at $525 \mathrm{MHz}$ induces current at the probe. Highlighting that the signal of both channels is in opposite phase, thus, as the EField is related with the subtraction of the data and the H-Field is correlated with the addition, it is clearly observed that the probe set-up corresponds for a maximum reception of the EField and minimum H-field. Finally, the time-domain data is subtracted in time-domain and then the FFT is applied, relating the value of voltage computed with the $5 \mathrm{~V} / \mathrm{m}$ incident E-Field to obtain the double-loaded loop probe factor (Fig. 3).
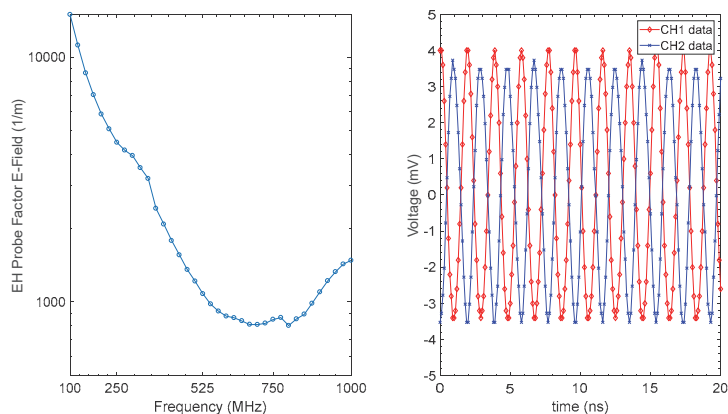

Fig. 3. Double-loaded probe factor (left) and time-domain data received by the oscilloscope when the $5 \mathrm{~V} / \mathrm{m} @ 525 \mathrm{MHz}$ electric field propagates through the $\mathrm{EH}$ probe at the $\mathrm{FAC}$ (right).

As it has been mentioned before, for validating and characterizing the developed EH-Field probe this procedure is sufficient. Nevertheless, if the objective is to calibrate the probe, a more exhaustive procedure is needed and the budget uncertainty is also required to estimate the accuracy of the double-loaded probe.

\section{UAV MEASUREMENTS APPLICATION}

In this section, the tailor-made probe is employed within the fuselage of a UAV measuring the electric field coupled at the interior of the cavity. Furthermore, with the aim to evaluate the response of the developed probe, we also conduct measurements with commercial probes and study the results through validation methods.

\section{A. Measurement scenario and test calibration}

The part of the UAV evaluated corresponds to the central fuselage of the INTA MILANO. The whole vehicle is $8.2 \mathrm{~m}$ long and $12.5 \mathrm{~m}$ wingspan but the part employed in this work is $3.5 \mathrm{~m}$ long and has a maximum height of $0.81 \mathrm{~m}$. The test is performed in an Open Area Test Site (OATS) with a concrete floor at INTA facilities in Madrid, Spain. The procedure is based on generating an electric field of $5 \mathrm{~V} / \mathrm{m}$ in a single point at $117 \mathrm{~cm}$ high from the floor and $3 \mathrm{~m}$ from the antenna. The polarization of the antenna is vertical to reduce the influence of possible ground floor reflections. The instrumentation to produce the electric field is a double ridge guide EMCO antenna model 3106 with a frequency range from $200 \mathrm{MHz}$ to $2 \mathrm{GHz}$, a PRANA amplifier model MT100S from $80 \mathrm{MHz}$ to 1 $\mathrm{GHz}$, and a HAMEG RF generator model HM8134. The electric field is measured by the reference AR laser-powered EField probe to reach the target field at the single calibrationpoint. All the instrumentation is controlled by a laptop via GPIB using MATLAB software. The frequency range of the test has been defined between $200 \mathrm{MHz}$ and $1 \mathrm{GHz}$, with a frequency step of $10 \mathrm{MHz}$; considering the bandwidth limitations of the transmitting antenna and the amplifier. After the E-Field is calibrated, the AR probe is substituted by the developed EH-Field probe described in section II.A. With this set-up the electric field is generated according to the previous calibration at the OATS with the tailor-made probe connected by means of a $50 \mathrm{~m}$ FO link to the Tektronix DPO 5104B oscilloscope.

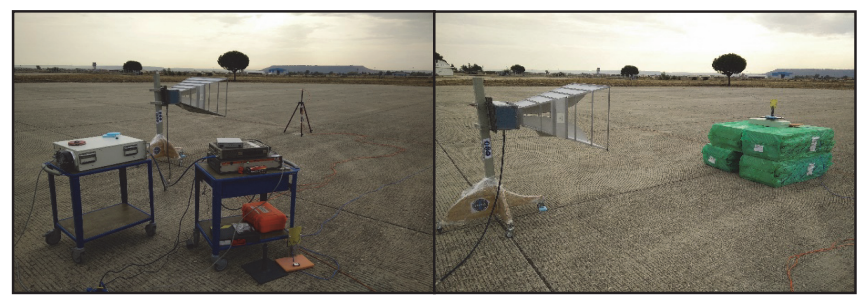

Fig. 4. OATS calibration and free space measurement

The results of the OATS measurement using the AR reference probe and the EH-Field probe are shown in Fig. 5. As it is observed, the total electric field (black trace) matches with the $z$ component (red trace), which is related with the vertical polarization of the electric field, on the other hand, the $x$ and $y$ components are negligible. Regarding the results obtained with the developed probe (blue trace), the measurement is according to the target pre-calibrated field obtaining a maximum 
deviation close to $0.5 \mathrm{~V} / \mathrm{m}(0.8 \mathrm{~dB})$, which is equal to the accuracy of the reference probe. Therefore, the results obtained with the constructed probe are successful and allows us to go on with the INTA MILANO measurements.

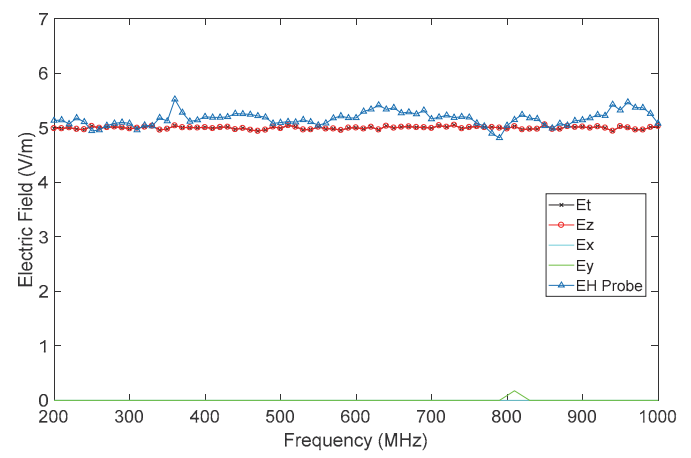

Fig. 5. Free space results for the AR reference probe and the EH probe

\section{B. INTA MILANO internal E-Field results}

The next step is to place the UAV at $3 \mathrm{~m}$ distance from the antenna, where the electric field has been previously calibrated. In this occasion, three different probes will be used to measure the E-Field within the composite material resonant-cavity of the INTA MILANO (Fig. 6). The probes used are the AR reference probe, the EH-Field probe developed and also a Ddot. The D-dot probe has large metallic parts and cables that introduce measurement uncertainty due to the modification of the fields. On the other hand, it is interesting to use the D-dot to compare our EH-Field probe with another commercial probe and to identify the differences between them caused by the placement of the different sensors.

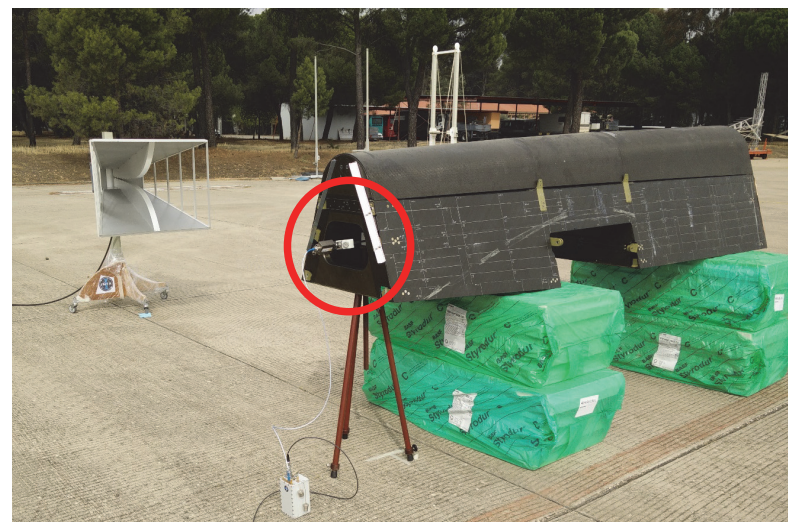

Fig. 6. INTA MILANO test set-up view when the D-dot is placed inside the fuselage

The first results are obtained with the AR reference E-Field probe, allocated at $117 \mathrm{~cm}$ high, which is the probe with a lower size and allows us to measure the three components of the E-Field simultaneously. In Fig. 7, it is observed that although the polarization of the field is vertical, inside the composite fuselage the E-Field has significant values at the three-axis. The EM wave is entering to the fuselage through the many apertures of the structure and fields are distributed and reflected inside the resonant cavity.

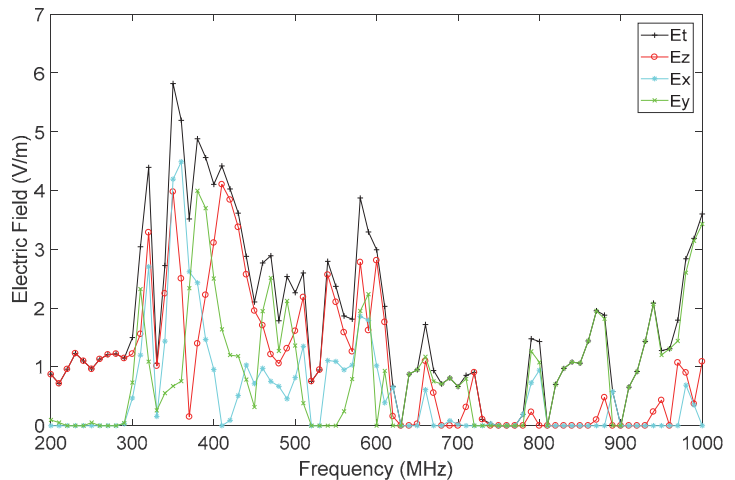

Fig. 7. Three-axes measurements of the E-Field inside the INTA Milano cavity using the AR probe

In order to compare the results obtained with the other probes, only the vertical component of the E-Field will be taken into consideration (z-axis). Other EMF components are remarkable, however this first-approach of the developed probe only measures one component of the E-Field simultaneously. Further development and investigation will be done to measure all the EMF components with a three-loop probe. Hence the Ddot and the EH-Field developed probe are mounted to measure the vertical component of the field as it can be seen in Fig. 8 for the time-domain double-loaded loop probe.

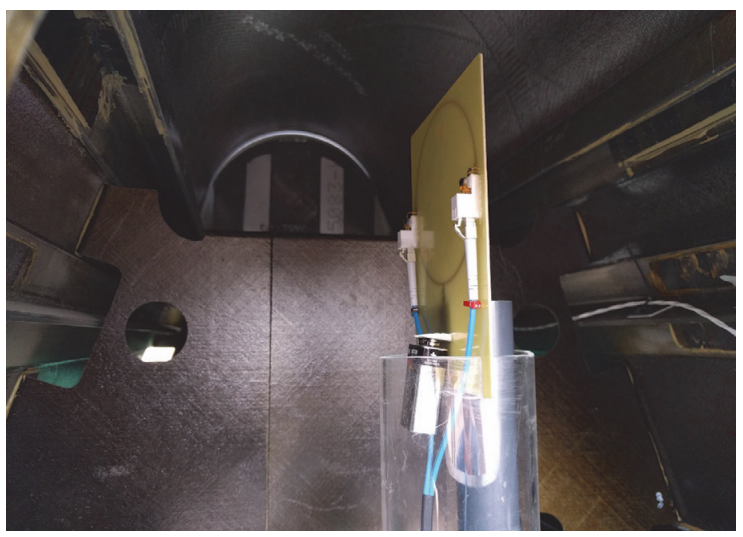

Fig. 8. EH-Field probe measuring the vertical component of the E-Field within the composite fuselage of the INTA Milano

Otherwise, another measurement at $110 \mathrm{~cm}$ high has also been done to try to identify changes of the E-Field when variations of few centimeters are introduced; simulating different sizes of the probes. The results are shown in Fig. 9. In red trace, we have our reference measurement carried out with the AR probe at $117 \mathrm{~cm}$ high, with the blue trace we plot the EField vertical component measured with the EH-Field developed probe, in green trace we show the D-dot result and in orange the result of the AR probe varying slightly the height to $110 \mathrm{~cm}$.

By examining the data, there seems to be a good agreement between the measurements employing the different probes. The values of the electric field level obtained along the $200 \mathrm{MHz}-1$ $\mathrm{GHz}$ frequency range are close to all the measurement systems. Identifying the frequency with the maximum field, all the 
significant resonances, and the frequency-ranges where E-Field amplitude is irrelevant. The frequency with the highest field level is $410 \mathrm{MHz}$ and above $600 \mathrm{MHz}$ the field within the composite material cavity is negligible. Between $300 \mathrm{MHz}$ and $500 \mathrm{MHz}$, where the maximum coupling is present, the developed probe measures all the peaks with a very good accuracy. The highest discrepancy is around $410 \mathrm{MHz}$ when the results are compared with the reference probe at $117 \mathrm{~cm}$; nevertheless, when the reference AR probe is placed with a minimum high variation $(110 \mathrm{~cm})$ the value of the electric field is perfectly matched. The reason of this difference is attributable to the size of the EH-Field probe, which is $10 \mathrm{~cm}$. Hence, the size of the developed probe seems to be a key factor when we are measuring within the resonant cavity. Finally, a higher difference appears between $600 \mathrm{MHz}$ and $700 \mathrm{MHz}$ when the D-dot is the measuring system inside the UAV. This can be associated with the size of the D-dot and the introduction of a coaxial cable inside the structure as it can be seen in Fig. 6.

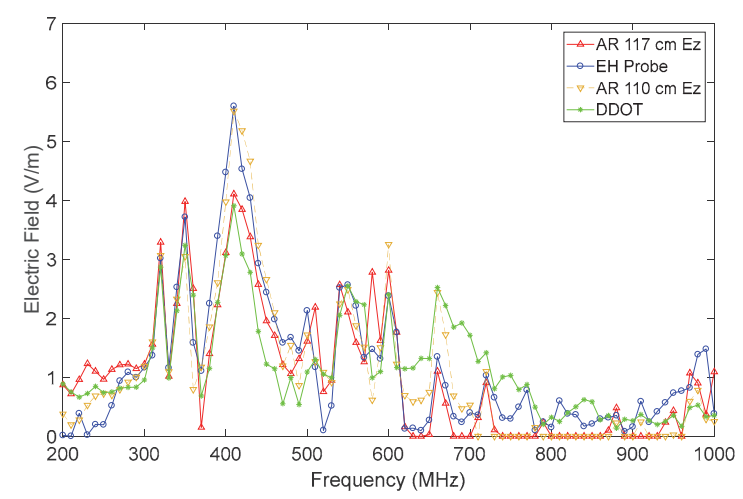

Fig. 9. Measurements conducted inside the fuselage of INTA MILANO employing the reference probe at two different heights, the D-dot and the EH probe.

Nevertheless, with the aim of validating the results obtained by the developed probe with the Full Time Domain measurement system, an objective evaluation is necessary. In next section, validation methods are applied to provide us quantitative values indicating if the E-Field results are accurate in all the frequency range in terms of amplitude and shape.

\section{Objective evaluation employing FSV and other validation methods}

Validation methods are necessary to objectively evaluate the similarity between different data sets in a qualitative way. Nowadays, several validation methods are available for test engineers to discuss if the data is confident with a requirement. Validation is usually carried out through comparison of a pattern or reference model with the model under study. Currently, one of the most used validation methods in the EMC field, because of its versatility and simplicity, is the Feature Selective Validation method (FSV) [15-17]. FSV, which is incorporated into IEEE standard 1597.1 [15], has the advantage of analyzing the two major aspects that are widely considered to be paramount in any validation: the magnitude levels and the shape of the dataset graphs.
Regarding the FSV results provided by the method, the data is compared in two diverse ways: the difference in amplitude (Amplitude Difference Measure, ADM) and the difference between the shape of the signal (Feature Difference Measure, FDM). In addition, a Global Difference Measure (GDM) is obtained from the ADM and FDM indicators, providing a global measurement of the overall difference. These indicators are very useful as they allow us to quickly evaluate the quality of the results as a single number is provided. The indicators are usually represented by histograms that are sorted according to the quality of the results into the following comparison categories: Excellent, Very Good, Good, Fair, Poor and Very Poor. Complementary to the FSV, other validation methods like the Integrated Error against Logarithmic Frequency (IELF) [18] have been applied for a better objective comparison. All the validations results have been obtained using the Global Validation Tool (GVT) developed by GCEM-UPC, which can be download from their website [19].

TABLE I. VALIDATION METHODS RESULT COMPARING THE RESULTS WITH THE REFERENCE AR PROBE AT 117 CM HIGH

\begin{tabular}{|l|c|c|c|c|c|}
\hline \multirow{2}{*}{ Probe } & \multicolumn{6}{|c|}{$\begin{array}{c}\text { Results of validation method compared to the reference AR } \\
\text { placed at 117 cm high }\end{array}$} \\
\cline { 2 - 6 } & FSV & IELF & Correlation & R Factor & $\begin{array}{c}\text { Diff. } \\
\text { Point-to- } \\
\text { point }\end{array}$ \\
\hline $\begin{array}{l}\text { EH } \\
\text { probe }\end{array}$ & $\begin{array}{c}0,51 \\
\text { Good }\end{array}$ & $\begin{array}{c}0.36 \\
\text { Excellent }\end{array}$ & $\begin{array}{c}0.91 \\
\text { Excellent }\end{array}$ & $\begin{array}{c}0.77 \\
\text { Excellent }\end{array}$ & $\begin{array}{c}0.30 \\
\text { Excellent }\end{array}$ \\
\hline $\begin{array}{l}\text { D-dot } \\
\text { Good }\end{array}$ & $\begin{array}{c}0.39 \\
\text { Excellent }\end{array}$ & $\begin{array}{c}0.79 \\
\text { Good }\end{array}$ & $\begin{array}{c}1.09 \\
\text { V. Good }\end{array}$ & $\begin{array}{c}0.40 \\
\text { Excellent }\end{array}$ \\
\hline $\begin{array}{l}\text { AR } \\
\text { probe } \\
110 \mathrm{~cm}\end{array}$ & $\begin{array}{c}0.6 \\
\text { Poor }\end{array}$ & $\begin{array}{c}0.38 \\
\text { Excellent }\end{array}$ & $\begin{array}{c}0.88 \\
\text { V. Good }\end{array}$ & $\begin{array}{c}1.07 \\
\text { V. Good }\end{array}$ & $\begin{array}{c}0.32 \\
\text { Excellent }\end{array}$ \\
\hline
\end{tabular}

The results presented in TABLE I show a great agreement between the AR reference probe and the developed EH-Field probe. The results are between good and excellent using five different objective validation methods, including the FSV standard. In conclusion, we can impartially determine that the double-loaded tailor-made loop probe produces confident results for the E-Field. Moreover, the results are more accurate than the ones obtained with the D-dot according to the different validation methods results. As it has been mentioned before, the placement of the D-dot and the measuring coaxial cables within the resonant cavity impacts on the accuracy of the results. Otherwise, it is also noticeable that varying the height of the AR reference probe just $7 \mathrm{~cm}$ at the interior of the fuselage produces deviations up to $1.5 \mathrm{~V} / \mathrm{m}$. Making that the agreement between the two measurements of the AR probes is lower than the agreement between the AR probe and the EHField probe or the D-dot according to the FSV results.

\section{CONCLUSIONS}

It has been demonstrated that it is suitable to employ the double-loaded developed loop probe with sufficient accuracy for measuring EMF within the composite resonant cavity of a UAV. Objective validation methodologies, such as FSV or IELF, have shown that the agreement between the measurements of the probe presented in this paper and the reference probe is good-excellent. Moreover, the EH-Field 
probe provides us other capabilities like measuring single frequencies in comparison with the traditional broadband EField probes. The minimized electronics at the probe, only considering the PCB track, the battery and the lasers contribute to a minimum distortion of the EMF. These advantages are referred to the availability of the full Time-domain method using the oscilloscope, having ready the time-domain acquired data to use powerful post-processing techniques. This is the main difference compared with the previously double-loaded loop probes, where the addition and the subtraction were done at the probe electronics. Otherwise, future studies should consider lower and higher frequency bands because one of the main applications is measuring impulsive broadband noise. Therefore, the size of the double-loaded loop has to be increased to gain sensibility at lower frequencies and the impact over the high-frequency measurement accuracy has to be analyzed.

Finally, other applications are possible as the time-domain data is available, for instance, the possibility of measuring high electromagnetic pulses or the electric and magnetic fields simultaneously at the three-axis. We conclude that the developed probe is an excellent approach for specific and tailor-made applications.

\section{ACKNOWLEDGMENT}

This work was supported in part by the EURAMET 15RPT01 research project (the EMPIR is jointly funded by the EMPIR participating countries within EURAMET and the European Union) and by the Spanish "Ministerio de Economía y Competitividad," under project TEC2016-79214-C3-\{1,2,3\}R (AEI/FEDER, UE).

\section{REFERENCES}

[1] M. R. Cabello et al., "SIVA UAV: A Case Study for the EMC Analysis of Composite Air Vehicles," in IEEE Transactions on Electromagnetic Compatibility, vol. 59, no. 4, pp. 1103-1113, Aug. 2017.

[2] S. G. Garcia et al., "UAVEMI project: Numerical and experimental EM immunity assessment of UAV for HIRF and lightning indirect effects," 2016 ESA Workshop on Aerospace EMC (Aerospace EMC), Valencia, 2016, pp. 1-5.

[3] X. Kong, Y. Z. Xie, Q. Li, S. Y. He and Y. B. Jin, "Development of One-Dimensional Norm Detector for Nanosecond-Level Transient Electric Field Measurement," in IEEE Transactions on Electromagnetic Compatibility, vol. 59, no. 4, pp. 1035-1040, Aug. 2017.

[4] M. A. Azpúrua, M. Pous and F. Silva, "On-board compact system for full time-domain electromagnetic interference measurements," 2016 ESA Workshop on Aerospace EMC (Aerospace EMC), Valencia, 2016, pp. 1-4.
[5] M. A. Azpúrua, M. Pous, S. Çakir, M. Cetinta and F. Silva, "Improving time-domain EMI measurements through digital signal processing," in IEEE Electromagnetic Compatibility Magazine, vol. 4, no. 2, pp. 82-91, 2nd Quarter 2015.

[6] R. W. P. King and C. Harrison, Jr., Antennas and Waves: A Modern Approach. Cambridge, UK: MIT Press, 1969.

[7] M. Kanda, "An electromagnetic near-field sensor for simultaneous electric and magnetic field measurements," IEEE Transactions on Electromagnetic Compatibility, vol. 26, no. 3, pp. 102-110, Aug. 1984.

[8] D. J. Bem and T. W. Wieckowsky, "Probes for EM pulse measurements," in Proc. 8th Int. Conf. EMC, London, U.K., 1992, pp. $125-128$.

[9] F. Silva, F. Sanchez, P. J. Riu and R. Pallas-Areny, "Low-cost near-field probe for simultaneous E and $\mathrm{H}$ measurement with analog optical link," IEEE 1997, EMC, Austin Style. IEEE 1997 International Symposium on Electromagnetic Compatibility. Symposium Record (Cat No.97CH36113), Austin, TX, 1997, pp. 533-536.

[10] M. Quílez, M. Aragón, A. Atienza, M. FernÁndez-Chimeno, P. J. Riu and F. Silva, "A Near-Field Probe for In Situ EMI Measurements of Industrial Installations," in IEEE Transactions on Electromagnetic Compatibility, vol. 50, no. 4, pp. 1007-1010, Nov. 2008.

[11] A. Atienza, M. Aragon, M. Quilez and F. Silva, "Electric and magnetic near-field measurements in industrial environments," 2008 International Symposium on Electromagnetic Compatibility - EMC Europe, Hamburg, 2008, pp. 1-4.

[12] M. A. Azpúrua, J. A. Oliva, M. Pous and F. Silva, "Fast and automated verification of multi-channel full time-domain EMI measurement systems," 2017 IEEE International Instrumentation and Measurement Technology Conference (I2MTC), Turin, 2017, pp. 1-6.

[13] M. Pous, M. Azpúrua and F. Silva, "Benefits of full time-domain EMI measurements for large fixed installation," 2016 International Symposium on Electromagnetic Compatibility - EMC EUROPE, Wroclaw, 2016, pp. 514-519.

[14] M. A. Azpúrua, M. Pous and F. Silva, "On the Statistical Properties of the Peak Detection for Time-Domain EMI Measurements," in IEEE Transactions on Electromagnetic Compatibility, vol. 57, no. 6, pp. 13741381, Dec. 2015.

[15] Standard for Validation of Computational Electromagnetics Computer Modeling and Simulations, IEEE Standard 1597.1.

[16] A. P. Duffy, A. J. M. Martin, A. Orlandi, G. Antonini, T. M. Benson, and M. S. Woolfson, "Feature selective validation (FSV) for validation, of computational electromagnetics (CEM). Part I-The FSV method," IEEE Trans. Electromagn. Compat., vol. 48, no. 3, pp. 449-459, Aug. 2006.

[17] R. Jauregui, M. Aragon, and F. Silva, "The role of uncertainty in the feature selective validation (FSV) method," IEEE Trans. Electrom. Compat., vol. 55, no. 1, pp. 217-220, Feb. 2013.

[18] R. J. Simpson, C. R. Jones, I. MacDiarmid, A. Duffy, and D. Coleby, "The integrated error against log frequency (IELF) method for CEM validation," in Electromagnetic Compatibility, 2005. EMC 2005. 2005 International Symposium on, 2005, vol. 1, p. 296--300 Vol.1.

[19] R. Jauregui, O. Ventosa, M. Pous and F. Silva, "GCEM validation tool (GVT)," 2018. [Online]. Available: http://www.upc.edu/web/gcem/?p=392\&lang=en 